\title{
First-principles calculation of current density in molecular devices
}

\author{
Lei Zhang, Bin Wang, and Jian Wang* \\ Department of Physics and the Center of Theoretical and Computational Physics, The University of Hong Kong, \\ Pokfulam Road, Hong Kong, China
}

(Received 8 April 2011; published 13 September 2011)

\begin{abstract}
Based on the single-particle nonequilibrium Green's function (NEGF) technique coupled with the densityfunctional theory (DFT), we investigate the current density distribution of a molecular device $\mathrm{Al}-\mathrm{C}_{60}-\mathrm{Al}$ from first principles. Due to the presence of nonlocal pseudopotential, the conventional definition of current density is not suitable to describe the correct current density profile inside the molecular device. By using the new definition of current density, which includes the contribution due to the nonlocal potential, our numerical results show that the new definition of current density $\mathbf{J}(\mathbf{r})$ conserves the current. In addition, the current obtained from the current density calculated inside the molecular device equals to that calculated from the Landauer-Büttiker formula. Finally, for the molecular device $\mathrm{Al}-\mathrm{C}_{60}-\mathrm{Al}$, loop currents were found, which confirms the result obtained from the tight-binding approach.
\end{abstract}

DOI: 10.1103/PhysRevB.84.115412

PACS number(s): 73.63.-b, 72.80.Rj, 85.65.+h, 73.23.Ad

\section{INTRODUCTION}

Due to the advance of nanofabrication technique, transport properties of molecular devices have attracted many research efforts both experimentally and theoretically. ${ }^{1-11}$ Experimentally, many interesting transport behaviors were found for molecular devices such as rectification, ${ }^{1}$ negative differential resistance, ${ }^{2}$ and conductance switching effects, ${ }^{3}$ which are very important from fundamental physics point of view and for their potential applications. On the theoretical side, based on the combination of single-particle nonequilibrium Green's function (NEGF) and density-functional theory (DFT), quantitative investigations ${ }^{10-13}$ have been carried out to explore the quantum transport properties of molecular devices from first principles. For instance, numerical investigation of current-voltage characteristics of molecular devices show negative differential resistance effect. ${ }^{14-16}$ In addition, other dc transport properties such as shot noise, ${ }^{17,18}$ thermoelectric properties, ${ }^{19}$ spin-dependent electron transport, ${ }^{20-22}$ and ac transport as well as transient transport ${ }^{23-27}$ of nanodevices have also been studied. Quantitative agreement between theoretical prediction and experimental results has been reached. For example, the current-voltage $(I-V)$ characteristic of the molecular wires made of oligophenylene molecules coupled with $\mathrm{Au}$ electrodes is found to be linear and the behavior of the small-bias resistances is increasing exponentially with an exponent $\beta$ as the wire length increases. The calculated value of $\beta$ is in quantitative agreement with experimental data. ${ }^{28}$

So far, most studies of these researchers mainly focus on the global transport property of molecular devices. It would be interesting to know what is the current flow pattern inside the molecular device. ${ }^{29,30}$ Because the current density profile can provide local quantum-transport information to analyze the structure properties and the heat generation, it can give us a vivid picture for a better understanding of the transport mechanism of the molecular device as well. It is the purpose of this paper to investigate the current density distribution of these systems. ${ }^{31}$

It is known that the current density is given by $\mathbf{J}_{c}(\mathbf{r})=$ $\frac{i e \hbar}{2 m}\left[\psi(\mathbf{r}) \nabla \psi^{*}(\mathbf{r})-\psi(\mathbf{r})^{*} \nabla \psi(\mathbf{r})\right]$ (when the magnetic field is zero), where $\psi$ is the scattering wave function. It is easy to show that the current obtained from the current density is conserved if the scattering potential is local, i.e., $V=V(\mathbf{r})$. For the first-principles investigation using density-functional theory, atomic cores are usually defined by the standard nonlocal norm-conserving pseudopotential. ${ }^{32}$ Furthermore, the exchange-correlation functional is a nonlocal function if we want to go beyond the LDA approximation, such as generalized-gradient approximation (GGA) ${ }^{33}$ As pointed out in Ref. 34, the conventional definition of current density does not conserve the current and hence does not give the correct current density in presence of the nonlocal potential. A new definition, detailed in Ref. 34, should be used in the presence of nonlocal pseudopotential. Since the current density has to be calculated in real space, it is difficult to implement the method proposed in Ref. 34 due to the memory problem, as will be discussed in detail in the next section. In this paper, we overcome the memory problem and use the new definition of current density $\mathbf{J}(\mathbf{r})$ to investigate the spatial distribution of current density inside the molecular device $\mathrm{Al}-\mathrm{C}_{60}-\mathrm{Al}$ from first principles. With the new definition, the current calculated from current density is, indeed, equal to that calculated from the Landauer-Büttiker formula. Our results show that the current density for the molecular device $\mathrm{Al}-\mathrm{C}_{60}-\mathrm{Al}$ is mainly distributed around each carbon atom and can form a circulating current around carbon atoms. As a further observation, we also find loop current in a hexagon face of the $\mathrm{C}_{60}$ molecule.

This paper is organized as follows. The theoretical formalism and detailed numerical techniques used in the calculation are discussed in Sec. II. In Sec. III, we present some numerical results to demonstrate our theoretical formalism. Section IV is the conclusion.

\section{THEORETICAL FORMALISM AND NUMERICAL TECHNIQUE}

By means of Keldysh formalism, the conventional definition of the current density in the absence of magnetic field can be expressed in terms of single-particle nonequilibrium Green's function: ${ }^{35}$

$$
\mathbf{J}_{c}(\mathbf{r})=-\frac{e \hbar}{2 m} \int \frac{d E}{2 \pi}\left[\left(\nabla-\nabla^{\prime}\right) G^{<}\left(\mathbf{r}, \mathbf{r}^{\prime}, E\right)\right]_{\mathbf{r}^{\prime}=\mathbf{r}},
$$


where $G^{<}=G^{r} \Sigma^{<} G^{a}$ is the lesser Green's function and $\Sigma^{<}=i \sum_{\alpha} \Gamma_{\alpha} f_{\alpha}$ is the corresponding self-energy due to the coupling to the lead. Here, $\Gamma_{\alpha}=i\left(\Sigma^{r}-\Sigma^{a}\right)$ is the linewidth function and $f_{\alpha}=f_{\alpha}\left(E-q V_{\alpha}\right)$ is the Fermi distribution function at $\alpha$ 's lead with the voltage $V_{\alpha}$ applied. In this formalism, the effect of semi-infinite leads is included by means of appropriate self-energies $\Sigma^{r, a}$. Therefore, once we know the Green's function in the central scattering region, the conventional current density $\mathbf{J}_{c}(\mathbf{r})$ can be calculated.

In order to investigate the current density $\mathbf{J}_{c}(\mathbf{r})$ inside the molecular device from first principles, we use the state-of-theart first-principles quantum-transport package MATDCAL, 36,37 which is based on the combination of NEGF and DFT theory (NEGF-DFT). Numerically, a linear combination of atomic orbitals (LCAO) is employed to solve the Kohn-Sham equations. The exchange-correlation is treated at the LDA level and the nonlocal norm-conserving pseudopotential is used to define the atomic core. The density matrix is constructed in orbital space and the effective potential is obtained in real space by solving the Poisson equation.

The NEGF-DFT self-consistency is reached when the numerical tolerance is less than $10^{-4} \mathrm{eV}$. Both self-consistent Hamiltonian and Green's function defined in orbital space are then obtained. Because the current density is defined in real space, a transformation from the orbital space to the real space should be made in order to calculate current density. According to the definition, the real-space lesser Green's function $G^{<}\left(\mathbf{r}, \mathbf{r}^{\prime}\right)$ can be expressed in terms of LCAO real-space basis set $\left\{\varphi_{\mu}(\mathbf{r})\right\}$

$$
G^{<}\left(\mathbf{r}, \mathbf{r}^{\prime}, E\right)=\sum_{\mu, \nu} \varphi_{\mu}(\mathbf{r}) G_{\mu \nu}^{<}(E) \varphi_{\nu}^{*}\left(\mathbf{r}^{\prime}\right),
$$

where $\mu$ and $v$ sum over all orbitals of the entire system and $G_{\mu \nu}^{<}(E)$ is Green's function defined in orbital space. Using the relation

$$
G^{<}=i G^{r} \Gamma G^{a} f_{R}+i G^{r} \Gamma_{L} G^{a}\left(f_{L}-f_{R}\right),
$$

the current density defined in Eq. (1) can be separated into equilibrium and nonequilibrium parts. Since the equilibrium part does not contribute to the transport current, ${ }^{34}$ we discuss only the second nonequilibrium part of the current density from now on. Furthermore, if we are only interested in the current density when the applied bias is small and the system is in the zero-temperature limit, the definition of conventional current density can be written as

$$
\mathbf{J}_{c}(\mathbf{r})=-\frac{i e^{2} \hbar}{4 \pi m}\left[\left(\nabla-\nabla^{\prime}\right) G^{n}\left(\mathbf{r}, \mathbf{r}^{\prime}, E_{f}\right)\right]_{\mathbf{r}^{\prime}=\mathbf{r}},
$$

with

$$
G^{n}\left(\mathbf{r}, \mathbf{r}^{\prime}\right) \equiv \sum_{\mu, \nu} \varphi_{\mu}(\mathbf{r})\left(G^{r} \Gamma_{L} G^{a}\right)_{\mu \nu} \varphi_{\nu}^{*}\left(\mathbf{r}^{\prime}\right) \delta V,
$$

where $\delta V=V_{L}-V_{R}$ is the voltage drop and $E_{f}$ is the Fermi level when the system is in equilibrium.

With the Green's function obtained for the molecular device, there are two ways of calculating the current. One can either calculate the current from Landauer-Büttiker formula or from the current density by integrating current density over any cross section inside the scattering region perpendicular to the transport direction (for instance, $S_{2}$ in Fig. 1). As discussed

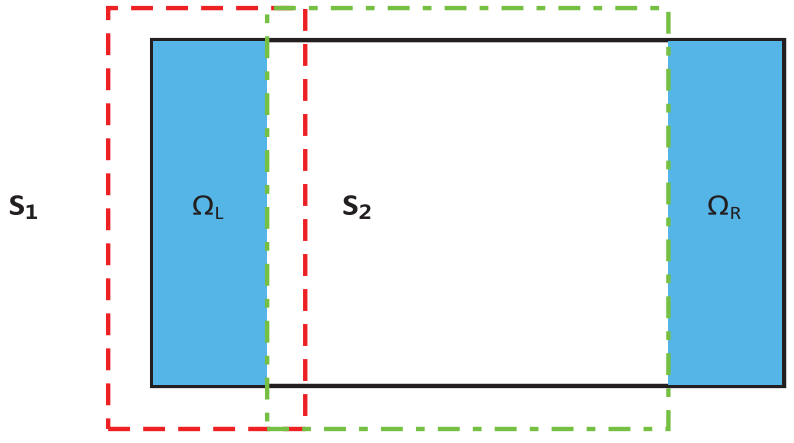

FIG. 1. (Color online) Schematic picture of the simulation box. The blue region $\Omega_{L} / \Omega_{R}$ is self-energy coupling regions due to the leads. The green dash-dot line encloses the central scattering region.

in detail in Ref. 34, the above conventional current density definition is not suitable to describe the correct current density if nonlocal potentials are present in the system. This is because the current obtained from the current density is not conserved, i.e., $\nabla \cdot \mathbf{J}_{c}=-\rho_{n} \neq 0$. Here, $\rho_{n}(\mathbf{r})$ is called nonlocal electron density due to the presence of nonlocal potential $V_{n l}\left(\mathbf{r}, \mathbf{r}^{\prime}\right)$ in the system, which is defined as

$$
\rho_{n}(\mathbf{r}, E)=\frac{i e^{2}}{h} \int d \mathbf{r}^{\prime}\left[V_{n l}\left(\mathbf{r}, \mathbf{r}^{\prime}\right) G^{n}\left(\mathbf{r}, \mathbf{r}^{\prime}, E_{f}\right)-\text { c.c. }\right] \text {. }
$$

To solve this problem, one can define a new current density $^{34}$

$$
\mathbf{J}(\mathbf{r}) \equiv \mathbf{J}_{c}(\mathbf{r})+\mathbf{J}_{n l}(\mathbf{r}),
$$

where $\mathbf{J}_{n l}(\mathbf{r})$ is defined as

$$
\mathbf{J}_{n l}(\mathbf{r})=-\nabla u(\mathbf{r}),
$$

with

$$
-\nabla^{2} u(\mathbf{r})=\rho_{n}(\mathbf{r})
$$

Therefore in order to calculate the nonlocal current density $\mathbf{J}_{n l}(\mathbf{r})$, we have to solve the Poisson equation (9) with proper boundary conditions once the nonlocal electron density $\rho_{n}(\mathbf{r})$
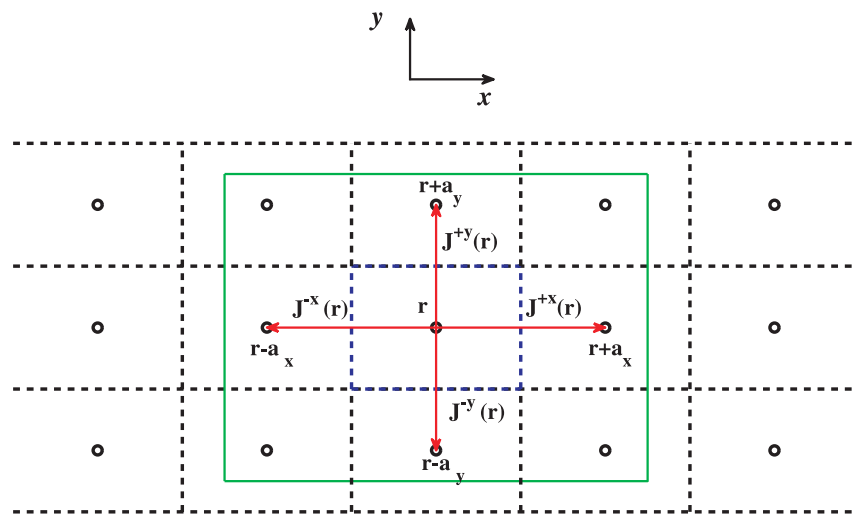

Left Lead

Central Region

Right Lead

FIG. 2. (Color online) A sketch illustrating current density defined between nearest-neighboring sites in a two-dimensional lattice model. Black circles represent lattice sites of the system. 


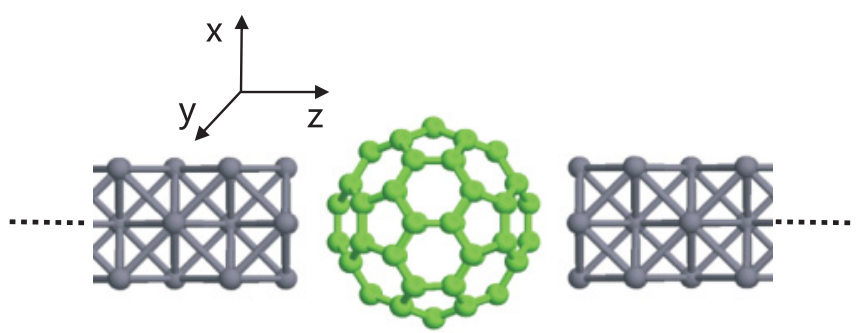

FIG. 3. (Color online) Schematic illustration of the $\mathrm{Al}-\mathrm{C}_{60}-\mathrm{Al}$ molecular device. The device consists of a $\mathrm{C}_{60}$ molecule coupled to the perfect aluminium atomic electrodes along (100) direction, which extend to the reservoirs at $\pm \infty$ where the current is collected.

is given. This simple scheme has been tested successfully in two-dimensional mesoscopic systems. ${ }^{34}$ However, it is very difficult to implement it directly to molecular devices because the nonlocal electron density in Eq. (6) is very hard to obtain in real space for two reasons. First of all, the number of grid points in real space is very large, on the order of a millon for a first-principles calculation of a simple molecular device. This means that $V_{n l}\left(\mathbf{r}, \mathbf{r}^{\prime}\right)$ is a huge nonsparse matrix, which can not be stored in the computer. Hence the memory availability is a big problem. Secondly, the basis set in orbital space is usually taken to be $s, p, d$, and $f$, which is not a complete basis set although it is sufficient enough to describe accurately the band structure as well as the transport properties. The consequence of this incompleteness is that even for a local potential $V(\mathbf{r})$, the transformation from orbital space to real space will eventually generate nondiagonal elements $\left.V\left(\mathbf{r}, \mathbf{r}^{\prime}\right)\right|_{\mathbf{r}^{\prime} \neq \mathbf{r}}$. This results in a large numerical error in the calculation of the nonlocal electron density $\rho_{n}(\mathbf{r})$.

An alternative way to avoid the above problems is to calculate the nonlocal electron density in orbital space and then transform it to real space. From Eqs. (7)-(9), we have

$$
\rho_{n}(\mathbf{r})=\nabla \cdot \mathbf{J}(\mathbf{r})-\nabla \cdot \mathbf{J}_{\mathbf{c}}(\mathbf{r}) .
$$

Note that the conventional current density can easily be calculated using Eqs. (4) and (5), so is its divergence $\nabla \cdot \mathbf{J}_{c}(\mathbf{r})$. The divergence of total current density $\mathbf{J}(\mathbf{r})$ can be calculated as follows:

$$
\begin{aligned}
\nabla \cdot \mathbf{J}(\mathbf{r}) & =\nabla \cdot \mathbf{J}_{c}(\mathbf{r})+\nabla \cdot \mathbf{J}_{n l}(\mathbf{r}) \\
& =-\frac{i e^{2} \hbar}{4 \pi m}\left[\left(\nabla^{2}-\nabla^{\prime 2}\right) G^{n}\left(\mathbf{r}, \mathbf{r}^{\prime}, E_{f}\right)\right]_{\mathbf{r}^{\prime}=\mathbf{r}}+\rho_{n},
\end{aligned}
$$

where we have used Eq. (4). From Eq. (6), we have

$$
\nabla \cdot \mathbf{J}(\mathbf{r})=\frac{i e^{2}}{h}\left[(H-E I) G^{n}-G^{n}(H-E I)\right]_{\mathrm{rr}} .
$$

To express Eq. (11) in orbital space, we use the definitions

$$
\begin{aligned}
H\left(\mathbf{r}, \mathbf{r}^{\prime}\right) & =\sum_{\mu, v} \tilde{\varphi}_{\mu}(\mathbf{r}) H_{\mu \nu} \tilde{\varphi}_{\nu}^{*}\left(\mathbf{r}^{\prime}\right), \\
I(\mathbf{r}, \mathbf{r}) & =\sum_{\mu, v} \tilde{\varphi}_{\mu}(\mathbf{r}) S_{\mu \nu} \tilde{\varphi}_{\nu}^{*}(\mathbf{r}),
\end{aligned}
$$

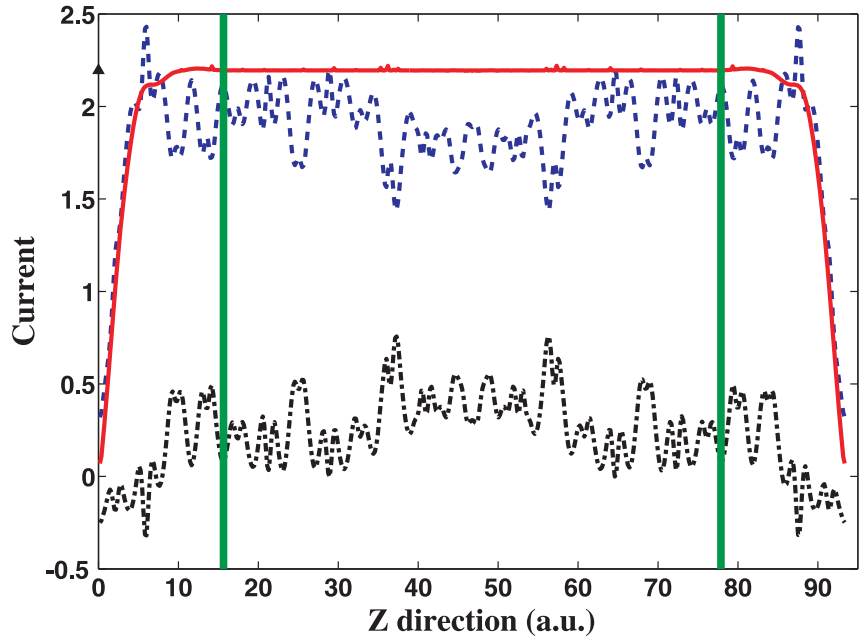

FIG. 4. (Color online) Depictions for the conventional current $I_{c}$ (blue dashed line), the nonlocal current $I_{n l}$ (black dash-dot line), and the total current $I$ (red solid line) along the transporting $Z$ direction. Here, the solid triangle on the Current axis indicates the current calculated from the Landauer-Büttiker formula and the green thick solid line is the boundary of the self-energy region.

where $I$ is the unit matrix, $S$ is the overlap matrix, and $\left\{\tilde{\varphi}_{\mu}(\mathbf{r})\right\}$ is the corresponding dual basis of $\left\{\varphi_{\mu}(\mathbf{r})\right\}$. Using Eq. (12), Eq. (11) becomes

$$
\begin{aligned}
\nabla \cdot \mathbf{J}(\mathbf{r})= & \frac{i e^{2}}{h} \sum_{\mu, \nu} \tilde{\varphi}_{\mu}(\mathbf{r})\left[(H-E S) G^{n}\right]_{\mu \nu} \varphi_{\nu}^{*}(\mathbf{r}) \\
& -\varphi_{\mu}(\mathbf{r})\left[G^{n}(H-E S)\right]_{\mu \nu} \tilde{\varphi}_{\nu}^{*}(\mathbf{r}) .
\end{aligned}
$$

Using the relation

$$
(E S-H) G^{r}=\left(I+\Sigma^{r} G^{r}\right),
$$

we have

$$
\begin{aligned}
\nabla \cdot \mathbf{J}(\mathbf{r})= & -\frac{i e^{2}}{h}\left\{\sum_{\mu, \nu} \tilde{\varphi}_{\mu}(\mathbf{r})\left[\left(I+\Sigma^{r} G^{r}\right) \Gamma_{L} G^{a}\right]_{\mu \nu} \varphi_{\nu}^{*}(\mathbf{r})\right. \\
& \left.-\varphi_{\mu}(\mathbf{r})\left[G^{r} \Gamma_{L}\left(I+G^{a} \Sigma^{a}\right)\right]_{\mu \nu} \tilde{\varphi}_{\nu}^{*}(\mathbf{r})\right\} \delta V .
\end{aligned}
$$

Note that because the basis set $\left\{\varphi_{\mu}(\mathbf{r})\right\}$ is not orthogonal, the corresponding dual basis set $\left\{\tilde{\varphi}_{\mu}(\mathbf{r})\right\}$ must be introduced as we did in the above discussion. To avoid calculating the dual basis, people usually use Gram-Schmidt orthonormalization to generate a set of orthogonal basis $\left\{\phi_{\mu}(\mathbf{r})\right\}$ based on the nonorthogonal basis $\left\{\varphi_{\mu}(\mathbf{r})\right\}$ so that the dual basis set is no longer needed. In this regards, the divergence of current density in the orthogonal orbital basis becomes

$$
\begin{aligned}
\nabla \cdot \mathbf{J}(\mathbf{r})= & -\frac{i e^{2}}{h} \sum_{\mu, v} \phi_{\mu}(\mathbf{r})\left[U\left(I+\Sigma^{r} G^{r}\right) \Gamma_{L} G^{a} U^{-1}\right. \\
& \left.-\left(U^{\dagger}\right)^{-1} G^{r} \Gamma_{L}\left(I+G^{a} \Sigma^{a}\right) U^{\dagger}\right]_{\mu \nu} \phi_{\nu}^{*}(\mathbf{r}) \delta V,
\end{aligned}
$$

where $\phi_{\mu}(\mathbf{r})$ is the corresponding orthogonal basis constructed from $\varphi_{\nu}(\mathbf{r})$ and $U$ matrix is defined as

$$
U_{\mu \nu}=\left\langle\phi_{\mu}(\mathbf{r}) \mid \tilde{\varphi}_{\nu}(\mathbf{r})\right\rangle .
$$



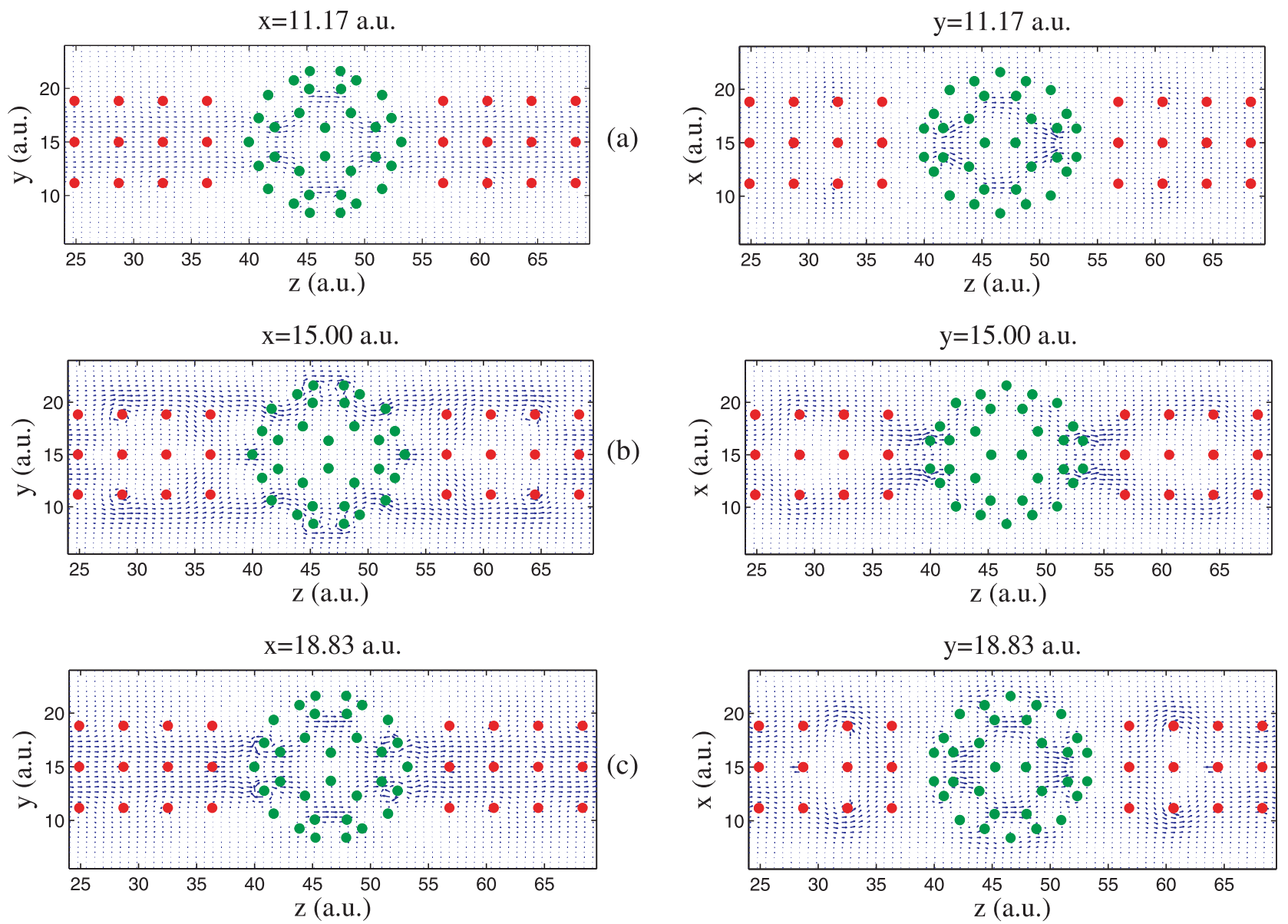

FIG. 5. (Color online) Distribution of the position-related current density $\mathbf{J}_{p}(\mathbf{r})$ (a)-(c) in $y$ - $z$ plane in several different $x$-axis slices and (d)-(f) in $x-z$ plane in several different $y$-axis slices. The arrows indicate the magnitude and direction of the current flow in each position. Red points represent $\mathrm{Al}$ atoms and green points represent $\mathrm{C}$ atoms projected onto the $y-z$ or $x-z$ plane.

In order to calculate the $U$ matrix, we define another auxiliary matrix:

$$
A_{\mu \nu}=\left\langle\varphi_{\mu}(\mathbf{r}) \mid \phi_{v}(\mathbf{r})\right\rangle
$$

and by using the orthogonality relation

$$
\delta_{\mu \nu}=\left\langle\phi_{\mu} \mid \phi_{\nu}\right\rangle=\sum_{i}\left\langle\phi_{\mu} \mid \tilde{\varphi}_{i}\right\rangle\left\langle\varphi_{i} \mid \phi_{\nu}\right\rangle=\sum_{i} U_{\mu i} A_{i \nu},
$$

$U$ can be calculated through the inversion of A matrix. Using Eqs. (4), (5), and (16), we can calculate the nonlocal electron density from Eq. (10).

Before we calculate the current density, we shall first prove that the current density defined in Eq. (7) gives the correct current that is calculated from Landauer-Büttiker formula. Let us consider a surface that encloses part of the scattering region with $\mathbf{S}_{\mathbf{1}}$ just outside the scattering region and $\mathbf{S}_{\mathbf{2}}$ the surface inside the central scattering region, as shown in Fig. 1. The volume $\Omega_{L}$ enclosed by this surface is such that $\Gamma_{L}$ is zero on surface $\mathbf{S}_{2}$. The current from the left lead is

$$
I_{L}=\int_{\mathbf{S}_{2}} \mathbf{J}(\mathbf{r}) \cdot d \mathbf{S}_{2}
$$

To show that this current is the same as that from LandauerBüttiker formula, we note that the current density is only defined inside the central region in the Keldysh formalism, which implies that the integration of $\mathbf{J}(\mathbf{r})$ over the surface $\mathbf{S}_{\mathbf{1}}$ outside the scattering region is zero. Then the current becomes

$$
I_{L}=\int_{\mathbf{S}} \mathbf{J}(\mathbf{r}) \cdot d \mathbf{S}=\int_{\Omega_{L}} \nabla \cdot \mathbf{J}(\mathbf{r}) d \mathbf{r} .
$$

From Eqs. (11) and (14) with the fact that $\Sigma^{r}=\Sigma_{L}^{r}$ in $\Omega_{L}$, we have

$$
\begin{aligned}
\operatorname{Tr}[\nabla \cdot \mathbf{J}(\mathbf{r})]_{\Omega_{L}}= & -\frac{i e^{2}}{h} \operatorname{Tr}\left[\left(I+\Sigma_{L}^{r} G^{r}\right) \Gamma_{L} G^{a}\right. \\
& \left.-G^{r} \Gamma_{L}\left(I+G^{a} \Sigma_{L}^{a}\right)\right]_{\Omega_{L}} \delta V .
\end{aligned}
$$

Since $\Sigma_{L}^{r}$ and $\Gamma_{L}$ are zero outside $\Omega_{L}$, the trace over $\Omega_{L}$ on the right-hand side of Eq. (22) equals to the trace over the whole scattering region $\Omega$, i.e., $\operatorname{Tr}[\cdots]_{\Omega_{L}}=\operatorname{Tr}[\cdots]_{\Omega}=$ $\operatorname{Tr}[\cdots]$. Finally, we have

$$
\begin{aligned}
I_{L} & =\operatorname{Tr}[\nabla \cdot \mathbf{J}(\mathbf{r})] \\
& =-\frac{i e^{2}}{h} \operatorname{Tr}\left[\Gamma_{L}\left(G^{a}-G^{r}\right)+\left(\Sigma_{L}^{r}-\Sigma_{L}^{a}\right) G^{r} \Gamma_{L} G^{a}\right] \delta V
\end{aligned}
$$



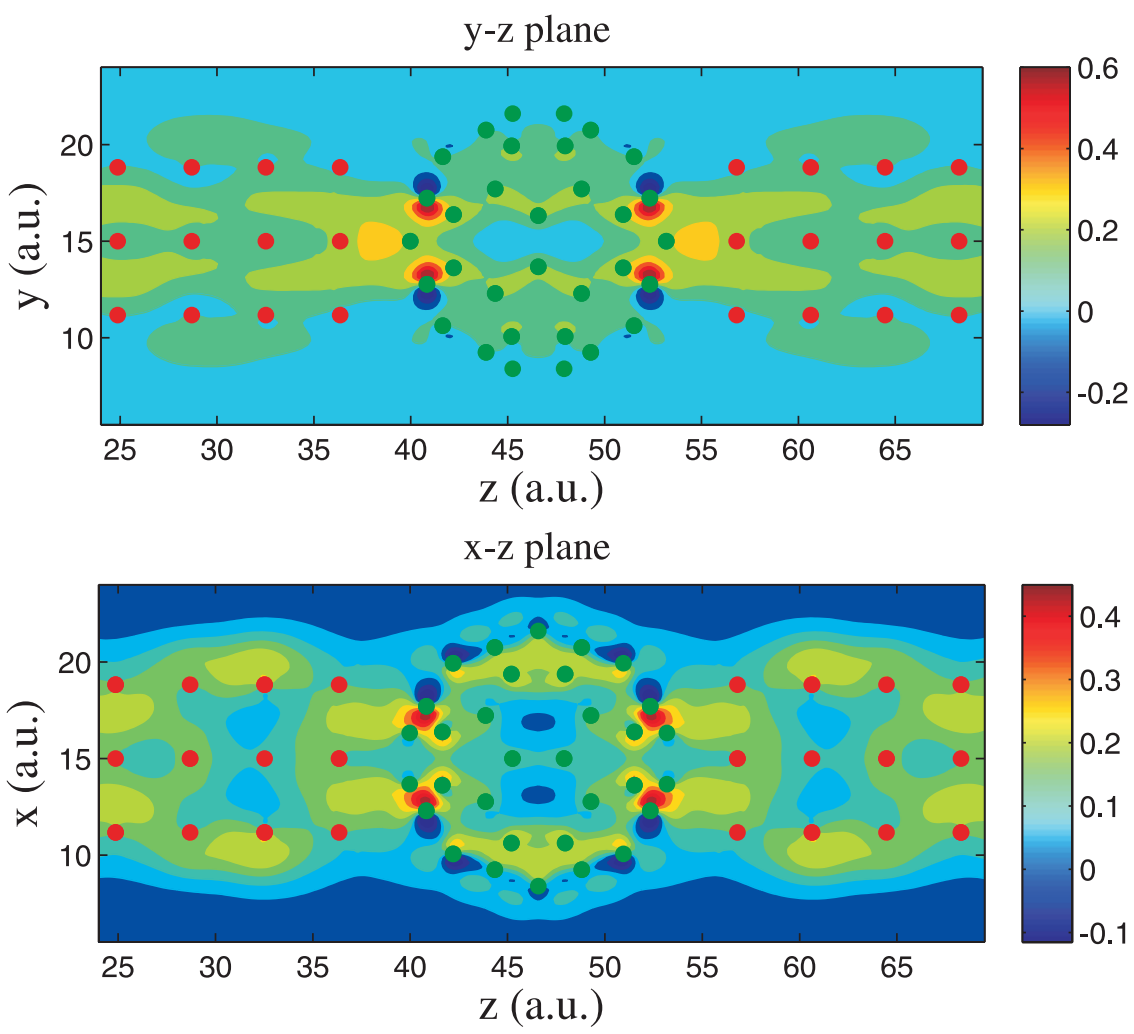

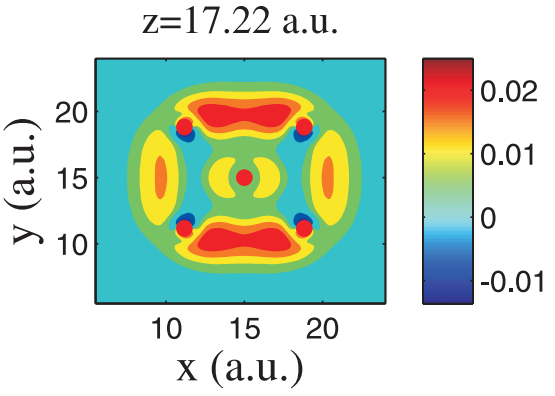

(c)

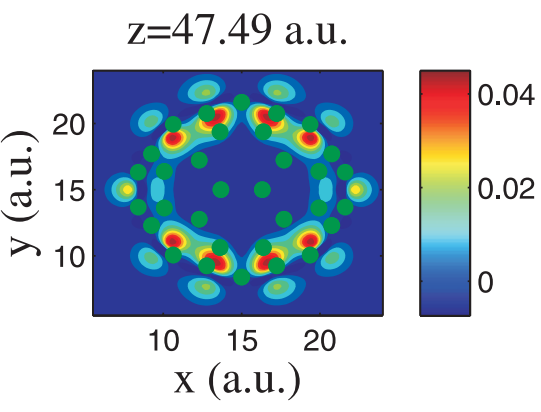

(d)

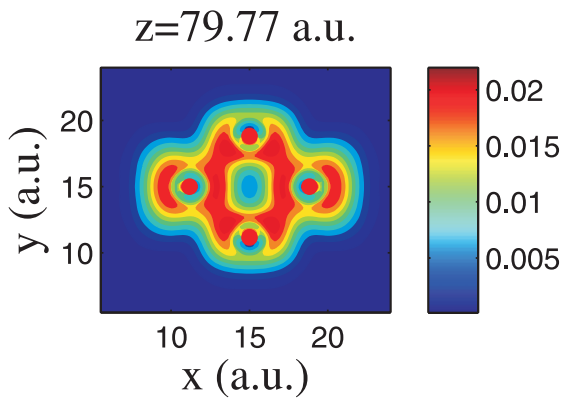

(e)

FIG. 6. (Color online) Contour plot of $z$ component of current density $\mathbf{J}(\mathbf{r})$. Red points represent $\mathrm{Al}$ atoms and green points represent $\mathrm{C}$ atoms projected onto the $x-z$ plane.

$$
\begin{aligned}
& =\frac{e^{2}}{h} \operatorname{Tr}\left[\Gamma_{L} G^{r} \Gamma G^{a}-\Gamma_{L} G^{r} \Gamma_{L} G^{a}\right] \delta V \\
& =\frac{e^{2}}{h} \operatorname{Tr}\left[\Gamma_{L} G^{r} \Gamma_{R} G^{a}\right] \delta V,
\end{aligned}
$$

where we have used the relation $G^{a}-G^{r}=i G^{r} \Gamma G^{a}$. Equation (23) is the same as that of the Landauer-Büttiker formula. This shows that as long as the surface $S_{2}$ is not inside the self-energy coupling regions $\Omega_{L}$ and $\Omega_{R}$, the integration of current density over $S_{2}$ is equal to the current given by the Landauer-Büttiker formula. In the actual calculation, we will choose the simulation box of the current density larger than the scattering region in which we are interested so that the current density is always conserved in the scattering region (see Fig. 1).

Now we discuss how to define the current density on a discrete lattice and the boundary condition of Poisson equation. For the purpose of discussion, we will illustrate our scheme in two dimensions (see Fig. 2). It is straightforward to generalize it to three dimensions. When solving a differential equation on a discrete lattice, the function itself such as $\rho(\mathbf{r})$ and $u(\mathbf{r})$ in Poisson equation and its second derivative $\nabla^{2} u(\mathbf{r})$ are defined on the grid point, while its first derivative such as the current density should be defined on the half grid point or on the bond connecting the two nearest-neighbor lattice sites. In this way, the continuity equation $\nabla \cdot \mathbf{J}(\mathbf{r})=0$ can be checked on each grid point inside the scattering region. In order to visualize the current density, one can define the current density on the grid point by taking the average over bond current density. From Eq. (4), the conduction current density flowing from site $\mathbf{r}$ to site $\mathbf{r} \pm a_{i}$ with $i=x, y$ can be defined as ${ }^{38}$

$$
J_{c}^{ \pm i}(\mathbf{r})=-\frac{i e^{2} \hbar}{4 \pi m a_{i}}\left[G^{n}\left(\mathbf{r} \pm a_{i}, \mathbf{r}\right)-G^{n}\left(\mathbf{r}, \mathbf{r} \pm a_{i}\right)\right]
$$


with $i=x, y$. The current density at the site $\mathbf{r}$ is obtained by averaging over the bond current connecting the site $\mathbf{r}$,

$$
J^{i}(\mathbf{r})=\frac{1}{2}\left[J^{+i}(\mathbf{r})-J^{-i}(\mathbf{r})\right],
$$

for $\mathbf{r}$ inside the scattering region. Because there is no current flowing from outside to the central scattering region, i.e., the bond current passing through the interface between the scattering region and outside is zero, we have to pay special attention to the boundary sites in defining the current density.

Concerning the boundary condition of Poisson equation, Eq. (9), since there is no current flowing into the scattering region, it is reasonable to assume that there is no nonlocal current density $J_{n l}^{i}(\mathbf{r})$ flowing out of the central region. This means that the boundary condition in solving the Poisson equation is the Neumann boundary condition where the normal derivative of the function $u(\mathbf{r})$ is specified on all boundaries, i.e., $\hat{\mathbf{n}} \cdot \mathbf{J}_{n l}(\mathbf{r})=\hat{\mathbf{n}} \cdot \partial u(\mathbf{r})=0$, where $\hat{\mathbf{n}}$ is the normal direction of the interface at the boundary.

To summarize the procedure of current density calculation, we first carry out ab initio calculations to obtain the Green's functions and potential landscape for a particular molecular device. We then calculate the conventional current density $\mathbf{J}_{c}(\mathbf{r})$ from Eqs. (4) and (5) and the nonlocal electron density according to Eqs. (10) and (16). Finally, the nonlocal current density $\mathbf{J}_{n l}(\mathbf{r})$ can be computed by solving the Poisson equation (9), and the total current density $\mathbf{J}(\mathbf{r})$ can be obtained.

\section{NUMERICAL RESULTS}

In this section, we implement our scheme and present the numerical results of current density of a molecular device: a $\mathrm{C}_{60}$ molecule coupled to two aluminum leads as shown in Fig. 3. For this system, there are 150 atoms in the central scattering region and 18 atoms in a unit cell with a finite cross section along (100) direction in the semi-infinite aluminum electrodes. In the $\mathrm{Al}-\mathrm{C}_{60}-\mathrm{Al}$ device, the distance between the $\mathrm{Al}$ atom and the nearest carbon atom is equal to $2.73 \mathrm{a}$.u. and the bond length of $\mathrm{C}-\mathrm{C}$ equals to 3.61 a.u. These distances as well as the entire device structure are relaxed under the equilibrium condition. In the calculation, the current is measured in the unit of $\left(e^{2} / h\right)$ volt, which means that we actually calculate $\mathbf{J}(\mathbf{r}) /(\delta V)$. Atomic units are adopted in the numerical calculations.

In order to demonstrate the current conservation, we denote the conventional current $I_{c}$ across the cross section located at $z$ as $I_{c}(z)=\int d x d y \widehat{\mathbf{z}} \cdot \mathbf{J}_{c}(x, y, z)$, and similarly, the nonlocal current $I_{n l}(z)=\int d x d y \widehat{\mathbf{z}} \cdot \mathbf{J}_{n l}(x, y, z)$ and the total current $I(z)=\int d x d y \widehat{\mathbf{z}} \cdot \mathbf{J}(x, y, z)$ of the system. In Fig. 4, we plot the conventional current $I_{c}$ and the nonlocal current $I_{n l}$ along the transport $z$ direction. Clearly, we can see that the conventional current varies at different cross sections along the transport $z$ direction. Most importantly, when the nonlocal current is included, the total current $I(z)$ obtained form the current density in the scattering region except the self-energy coupling region is a constant and equals to the current calculated from the Landauer-Büttiker formula (marked by a solid triangle in Fig. 4). This indicates that the current calculated from the new definition of current density in presence of the nonlocal potential is conserved. We also note that the total current is not conserved in the self-energy coupling region because the divergence of the current density $\mathbf{J}(\mathbf{r})$ is not equal to zero according to Eq. (15).

Since the current density is a vector field, we can visualize it by projecting the current density onto different slices along each direction ( $x$ or $y$ directions). In Fig. 5, we plot $x$ and $y$ components of position-related current density $\mathbf{J}_{p}(\mathbf{r})$ versus axis $x-y$ and $z$, where $\mathbf{J}_{p}(\mathbf{r})$ is defined as the sum of several neighboring points in the same plane, i.e., $J_{p}^{i}\left(\mathbf{r}^{\prime}\right)=$ $\sum_{j=x, y} \sum_{\mathbf{r}}^{\mathbf{r}+2 a_{j}} J^{i}(\mathbf{r})$ and $i=x-y$. As shown in Fig. 5, the current density is mainly distributed around each atom and is scattered due to the atomic potential. Note that for illustration purposes, we have shown all the atoms of $C_{60}$ in the plot by projection. But many atoms are not in the cutting plane so that the current density around those atoms is small. For instance, two atoms in the center of Fig. 5(b) are far away from the cutting plane so that the current density around these two atoms is very small. By comparing different panels in Fig. 5, we can clearly see that the current density distribution is different due to the effect of fullerene $\mathrm{C}_{60}$ when we cut different slices of the whole system. Because the Hartree potential near the carbon atoms is very different from that near the $\mathrm{Al}$ atoms, the local potential will redistribute the current density flow from the $\mathrm{Al}$ buffer layer to the fullerene $\mathrm{C}_{60}$ molecule. The distribution of current density due to the scattering from $\mathrm{Al}$ to $\mathrm{C}$ atoms can be clearly seen in Fig. 5 .

Since the $z$ component of current density dominates the transport properties of the molecular device, we show the contour plot of the current density $J^{z}(\mathbf{r})$ in Fig. 6, which reflects the local transport information of molecular device. In the $x-z$ and $y-z$ planes, we average all slices in $y$ and $x$ directions to get $\overline{J^{z}}(\mathbf{r})$. Furthermore, several slices in $z$ direction are chosen to visualize the current density $J^{z}(\mathbf{r})$ in $x-y$ plane. From Figs. 6(a) and 6(b), we see that the $z$ component of current density has positive and negative values around $z=41$ a.u showing circulating current around the

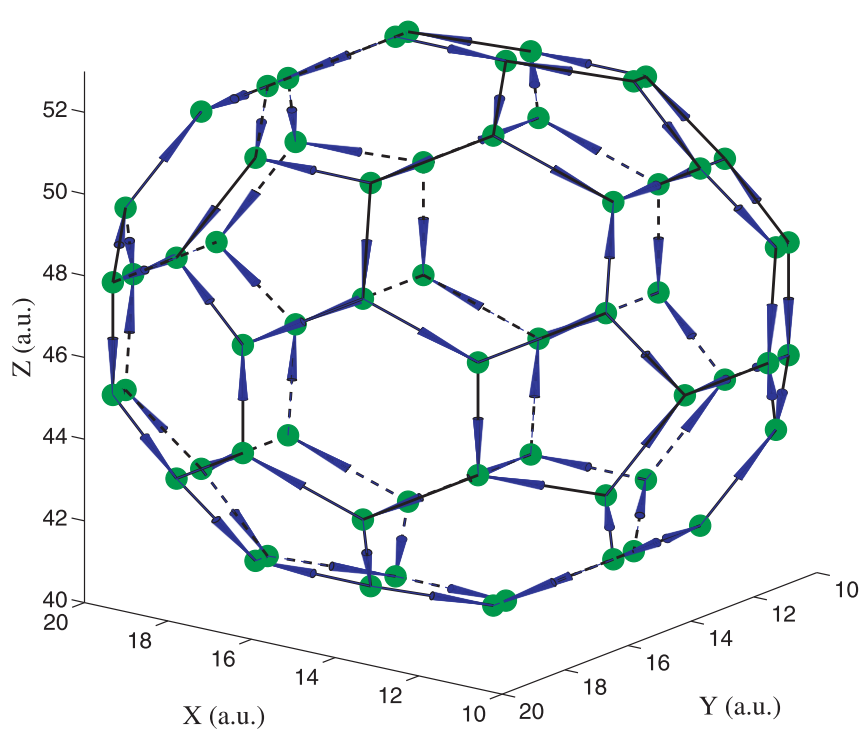

FIG. 7. (Color online) Current distribution of the fullerene $\mathrm{C}_{60}$ Green points represent carbon atoms and blue arrows point to the direction of the current flow from one atom to another. 
carbon atoms near the $\mathrm{Al}$ leads. It has been reported, ${ }^{39}$ using a tight-binding model, that there may be a loop current in the fullerene $\mathrm{C}_{60}$. To examine this prediction, we firstly define the current from atom $j$ to atom $i$, which can be calculated from the Green's function $G^{n}$ in Eq. (7): ${ }^{39}$

$$
I_{i j}\left(E_{f}\right)=\frac{4 e}{h} \sum_{l_{1}, l_{2}=s, p} \operatorname{Im}\left[H_{i_{l_{1}} j_{l_{2}}} G_{i_{l_{1}} j_{l_{2}}}^{n}\left(E_{f}\right)\right],
$$

where the summation of orbital $s$ and $p$ is taken over two atoms. Here, $H$ and $G^{n}$ are defined in orbital space in our first-principles calculation. Our result is shown in Fig. 7 where we do see the loop current although the current pattern is different from that of Ref. 39. We think this difference is due to the one-dimensional leads used in Ref. 39.

\section{SUMMARY}

In conclusion, the issue of current density conservation is addressed in the first-principles calculation. Due to the presence of nonlocal pseudopotential, the conventional current density in terms of the single-particle nonequilibrium Green's function is not appropriate to provide the correct information of current flow inside the molecular device. By introducing the nonlocal current density, the total current density $\mathbf{J}(\mathbf{r})$ satisfies the continuity equation, which means the current calculated from the total current density across an arbitrary cross section inside the central scattering region is a constant and equals to the current calculated from the Landauer-Büttiker formula. Details of how to implement this formalism for molecular devices are presented. As an application of our formalism, we have numerically calculated the current density for the Al- $\mathrm{C}_{60}-\mathrm{Al}$ molecular device. It is shown that the current calculated from current density is equal to that calculated from the Landauer-Büttiker formula, which numerically confirms our formalism to calculate current density from first principles. In addition, we verified that loop current, which can induce magnetic moment, also exists in a molecular device consisting of fulleren-e $\mathrm{C}_{60}$ coupled with two aluminium leads.

\section{ACKNOWLEDGMENTS}

We gratefully acknowledge the support from Research Grant Council (HKU 705409P) and University Grant Council (Contract No. AoE/P-04/08) of the Government of HKSAR. *jianwang@hkusua.hku.hk

${ }^{1}$ A. Aviram and M. Ratner, Chem. Phys. Lett. 29, 277 (1974).

${ }^{2}$ J. Chen, M. A. Reed, A. M. Rawlett, and J. M. Tour, Science 286, 1550 (1999).

${ }^{3}$ C. P. Collier et al., Science 285, 391 (1999).

${ }^{4}$ C. Joachim, J. K. Gimzewski, and A. Aviram, Nature (London) 408, 541 (2000).

${ }^{5}$ J. R. Heath and M. A. Ratner, Phys. Today 56, 43 (2003).

${ }^{6}$ J. R. Petta, S. K. Slater, and D. C. Ralph, Phys. Rev. Lett. 93, 136601 (2004).

${ }^{7}$ N. J. Tao, Nat. Nanotechnology 1, 173 (2006).

${ }^{8}$ M. Di Ventra, S. T. Pantelides, and N. D. Lang, Phys. Rev. Lett. 84, 979 (2000).

${ }^{9}$ J. Taylor, H. Guo, and J. Wang, Phys. Rev. B 63, 121104 (2001).

${ }^{10}$ J. Taylor, H. Guo, and J. Wang, Phys. Rev. B 63, 245407 (2001).

${ }^{11}$ M. Brandbyge, J. L. Mozos, P. Ordejon, J. Taylor, and K. Stokbro, Phys. Rev. B 65, 165401 (2002).

${ }^{12}$ S. H. Ke, H. U. Baranger, and W. T. Yang, Phys. Rev. B 70, 085410 (2004).

${ }^{13}$ Y. Q. Xue, S. Datta, and M. A. Ratner, Chem. Phys. 281, 151 (2002).

${ }^{14}$ C. C. Kaun, B. Larade, H. Mehrez, J. Taylor, and H. Guo, Phys. Rev. B 65, 205416 (2002).

${ }^{15}$ R. Pati, M. McClain, and A. Bandyopadhyay, Phys. Rev. Lett. 100, 246801 (2008).

${ }^{16}$ K. H. Bevan, D. Kienle, H. Guo, and S. Datta, Phys. Rev. B 78, 035303 (2008).

${ }^{17}$ Y. C. Chen and M. Di Ventra, Phys. Rev. B 67, 153304 (2003).

${ }^{18}$ J. Lagerqvist, Y. C. Chen, and M. Di Ventra, Nanotechnology 15, S459 (2004).

${ }^{19}$ B. Wang, Y. X. Xing, L. H. Wan, Y. D. Wei, and J. Wang, Phys. Rev. B 71, 233406 (2005).

${ }^{20}$ R. Pati, L. Senapati, P. M. Ajayan, and S. K. Nayak, Phys. Rev. B 68, 100407(R) (2003).
${ }^{21}$ Z. Y. Ning, Y. Zhu, J. Wang, and H. Guo, Phys. Rev. Lett. 100, 056803 (2008).

${ }^{22}$ H. Sahin and R. T. Senger, Phys. Rev. B 78, 205423 (2008).

${ }^{23}$ J. L. Wu, B. G. Wang, J. Wang, and H. Guo, Phys. Rev. B 72, 195324 (2005).

${ }^{24}$ S. Kurth, G. Stefanucci, C. O. Almbladh, A. Rubio, and E. K. U. Gross, Phys. Rev. B 72, 035308 (2005).

${ }^{25}$ X. Zheng, F. Wang, C. Y. Yam, Y. Mo, and G. H. Chen, Phys. Rev. B 75, 195127 (2007).

${ }^{26}$ B. Wang and J. Wang, Phys. Rev. B 77, 245309 (2008).

${ }^{27}$ B. Wang, Y. X. Xing, L. Zhang, and J. Wang, Phys. Rev. B 81, 121103 (2010).

${ }^{28}$ C. C. Kaun, B. Larade, and H. Guo, Phys. Rev. B 67, 121411 (2003).

${ }^{29}$ N. D. Lang, Phys. Rev. B 52, 5335 (1995).

${ }^{30}$ Y. Q. Xue and M. A. Ratner, Phys. Rev. B 70, 081404 (2004).

${ }^{31}$ Y. Liu and H. Guo, Phys. Rev. B 69, 115401 (2004).

${ }^{32}$ D. R. Hamann, M. Schlüter, and C. Chiang, Phys. Rev. Lett. 43, 1494 (1979).

${ }^{33}$ J. P. Perdew, J. A. Chevary, S. H. Vosko, K. A. Jackson, M. R. Pederson, D. J. Singh, and C. Fiolhais, Phys. Rev. B 46, 6671 (1992).

${ }^{34}$ C. S. Li, L. H. Wan, Y. D. Wei, and J. Wang, Nanotechnology 19, 155401 (2008).

${ }^{35}$ S. Datta, Electronic Transport in Mesoscopic Systems (Cambridge University Press, Cambridge, 1995).

${ }^{36}$ D. Waldron, P. Haney, B. Larade, A. MacDonald, and H. Guo, Phys. Rev. Lett. 96, 166804 (2006).

${ }^{37}$ D. Waldron, V. Timoshevskii, Y. Hu, K. Xia, and H. Guo, Phys. Rev. Lett. 97, 226802 (2006).

${ }^{38}$ H. Jiang, L. Wang, Q. F. Sun, and X. C. Xie, Phys. Rev. B 80, 165316 (2009).

${ }^{39}$ S. Nakanishi and M. Tsukada, Phys. Rev. Lett. 87, 126801 (2001). 